\title{
Screening for chlamydial infections and the risk of ectopic pregnancy in a county in Sweden: ecological analysis
}

\author{
Matthias Egger, Nicola Low, George Davey Smith, Bo Lindblom, Björn Herrmann
}

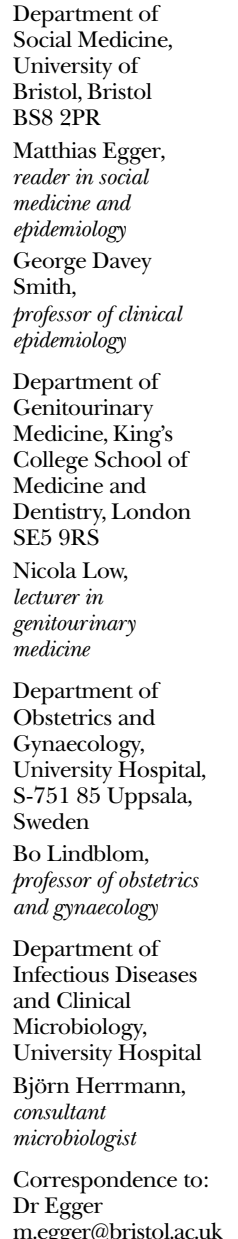

1998:316:1776-80

\begin{abstract}
Objectives: To analyse trends in rates of genital chlamydial infection and ectopic pregnancy between 1985 and 1995 in a county in Sweden.

Setting: Uppsala county where screening for chlamydial infection, treatment, and contact tracing has been widespread and where ectopic pregnancies are recorded.
\end{abstract}

Methods: Rates of chlamydial infections and ectopic pregnancy between 1985 and 1995 were calculated for women aged 20-39 years. Poisson and linear regression were used to examine the association between the risk of ectopic pregnancy and the current rates of chlamydial infection and rates of chlamydial infection from up to 5 years earlier.

Main outcome measures: Rates of chlamydial infection per 100 examinations, rates of ectopic pregnancy per 1000 pregnancies, rate ratios and 95\% confidence intervals for an increase in chlamydial infections of 5 new cases per 100 examinations. Results: 103870 cervical samples from women aged 15 -39 years were analysed; 5648 (5.4\%) were positive for chlamydia. 51630 pregnancies were analysed; 930 $(1.8 \%)$ were ectopic. Both rates declined over time. For women aged 20-24 years there was a strong correlation between the rate of ectopic pregnancy and the rate of chlamydial infection in the same year $(r=0.93, \mathrm{P}<0.001)$; among older women correlations were stronger with rates of chlamydial infection occurring 1 or 2 years earlier. In Poisson regression ectopic pregnancy was most strongly associated with the current rate of chlamydial infection among women aged 20-24 (rate ratio 1.85, 95\% confidence interval 1.44 to 2.38 ), with the rate of infection 1 year earlier among 25-29 year olds (rate ratio 1.72, 1.33 to 2.22 ) and $30-34$ year olds (rate ratio $2.27,1.53$ to 3.37); and with the rate 2 years earlier among 35 to 39 year olds (rate ratio $2.58,1.45$ to 4.60 ).

Conclusions: Declining rates of genital chlamydial infections have probably led to a fall in the rate of ectopic pregnancies. The timing of the decline in the rate of ectopic pregnancies varies with age. Among young women falling rates of chlamydial infection have been accompanied by an immediate reduction in the rate of ectopic pregnancy.

\section{Introduction}

In industrialised countries Chlamydia trachomatis is the predominant infectious agent causing pelvic inflammatory disease $\mathrm{e}^{1-3}$ and, as a result of damage to the fallopian tubes, accounts for up to half of all ectopic pregnancies. ${ }^{45}$ The substantial financial costs of genital chlamydial infections result from hospital treatment for pelvic inflammatory disease, ectopic pregnancy, and infertility, which may include in vitro fertilisation. ${ }^{6}$ Programmes to reduce the incidence of genital chlamydial infection have not been widely implemented except in Sweden.

Policies to prevent chlamydial infection were gradually introduced in the 1980 s in Sweden. ${ }^{7}$ These included the establishment of a national diagnostic service $^{8}$; testing of women in antenatal, family planning, and abortion clinics ${ }^{89}$; a statutory requirement since 1988 that doctors trace and treat the sexual contacts of patients with chlamydial infections ${ }^{10}$; and the establishment of youth clinics which provide health education, condoms, and testing and treatment for chlamydial infection. ${ }^{8}$

Declining rates of chlamydial infection and associated pelvic inflammatory disease in Sweden during the 1980 s have been attributed to these policies. ${ }^{811}$ The effect of these policies on the incidence of sequelae such as ectopic pregnancy and infertility are less clear. Trends in the occurrence of ectopic pregnancy are thought to lag behind those of chlamydial infection by several years, ${ }^{45}$ but this association has not been examined in a population based study. Uppsala county, north of Stockholm, consists of six municipalities with a total population of 269000 in 1990. Screening for chlamydial infection among the female population has been extensive ${ }^{8}$ and care for ectopic pregnancies is provided in one hospital only. Uppsala county thus provides an exceptional setting for an ecological study of the association between genital chlamydial infection and ectopic pregnancy.

\section{Material and methods}

\section{Ascertainment of infections and pregnancies}

The Uppsala chlamydia database has been described in detail elsewhere. ${ }^{8}$ Briefly, over $99 \%$ of examinations for infection with $C$ trachomatis are performed in a single laboratory. A total of 110834 cervical samples were collected from women of all ages between 1985 and 1995. Overall, $23 \%$ of samples came from 20 private practices, $16 \%$ from one sexually transmitted disease clinic, $15 \%$ from three family planning clinics, $14 \%$ from six youth clinics, $14 \%$ from five gynaecology clinics, $10 \%$ from 34 primary healthcare clinics, $7 \%$ from 14 antenatal clinics, and $1 \%$ from other sites. Altogether $89 \%(99133 / 110834)$ of samples were cultured in McCoy cells treated with cycloheximide, according to the methods of Ripa and Mårdh ${ }^{12} ; 10 \%$ (10 855/110 834) were analysed by enzyme immunoassay (EIA, Abbott Diagnostics, Chicago, or Syva, Palo Alto); and $0.8 \%(846 / 110834)$ were analysed by direct immunofluorescence (Syva, Palo Alto). Results of 568 examinations $(0.5 \%$ of all samples) could not be interpreted.

The list of discharge diagnoses from University Hospital in Uppsala was used to determine the number of times ectopic pregnancy was the primary diagnosis for women aged 15 to 39. This list includes all episodes diagnosed in the county. Population numbers, births, 
Table 1 Female population in Uppsala, Sweden, in 1990, chlamydia test and result, and number of pregnancies and their outcome, 1985-95, by age group. Values are numbers (percentages)

\begin{tabular}{|c|c|c|c|c|c|c|c|}
\hline \multirow[b]{2}{*}{ Age (years) } & \multirow[b]{2}{*}{ Population 1990} & \multicolumn{2}{|c|}{ Chlamydia tests } & \multirow[b]{2}{*}{ Pregnancies } & \multirow[b]{2}{*}{ Births } & \multirow[b]{2}{*}{ Induced abortions } & \multirow[b]{2}{*}{ Ectopic pregnancies } \\
\hline & & Total & Positive & & & & \\
\hline $20-24$ & 10021 & 39547 & $2590(6.5)$ & 12033 & $8480(70.5)$ & $3450(28.6)$ & $103(0.9)$ \\
\hline $25-29$ & 10650 & 22341 & $838(3.8)$ & 18372 & 15369 (83.7) & $2730(14.8)$ & $273(1.5)$ \\
\hline $30-34$ & 9659 & 9939 & $240(2.4)$ & 14354 & $11863(82.6)$ & $2162(15.1)$ & $329(2.3)$ \\
\hline $35-39$ & 9776 & 6490 & $146(2.2)$ & 6871 & $4936(71.7)$ & $1710(24.9)$ & $225(3.3)$ \\
\hline Total & 40106 & 78317 & $3814(4.9)$ & 51630 & 40648 (78.7) & 10052 (19.5) & $930(1.8)$ \\
\hline
\end{tabular}

and the number of induced abortions were obtained for women at each age from the county statistical office or the epidemiological centre of the National Board of Health and Welfare. The total number of pregnancies reported was calculated as the sum of live births, induced abortions, and ectopic pregnancies.

\section{Statistical analysis}

Rates of genital chlamydial infection per 100 examinations and rates of ectopic pregnancy per 1000 reported pregnancies between 1985 and 1995 were calculated for women aged 20-24, 25-29, 30-34, and 35-39 years. The association between the two rates was examined using linear regression analysis. $\chi^{2}$ tests were used to examine trends by age.

The same rates were calculated for women at each age and for each calendar year for chlamydial infection among women aged 15-39 and for ectopic pregnancy among those aged 20-39. Poisson regression models were defined to examine the strength of the association between the rates of chlamydial infection and the risk of ectopic pregnancy at each age. Models were calculated to examine the association between the rate of ectopic pregnancy and the rate of chlamydial infection in the same year and for the rates of chlamydial infection occurring 1 to 5 years earlier. For example, in the model examining the importance of the rate of chlamydial infection 5 years earlier, the rate among 15 year olds in 1985 was used to predict the risk of ectopic pregnancy among 20 year olds in 1990. This meant that some rates were missing in earlier years. The rate 1 year earlier was available from 1986 onwards and the rate five years earlier from 1990 onwards. Analyses were adjusted for age within each age group.

Results from Poisson regression models are given as rate ratios with $95 \%$ confidence intervals to estimate the effect of an increase in the rate of chlamydial infection of 5 new infections for every 100 examinations.

\section{Results}

Table 1 shows the number of chlamydia tests, reported pregnancies, and total female population during the study for women aged 20-39 years. The calculation of the rates of genital chlamydial infection in this age group was based on a total of 78317 samples of which $3814(4.9 \%)$ were positive. There were 51630 pregnancies of which $930(1.8 \%)$ were ectopic. Sixty three per cent $(32726 / 51630)$ of pregnancies occurred among women aged 25-34 years, and 50\% (39 547/78 317) of chlamydia tests were performed on women aged 20-24 years. Rates of chlamydial infection and rates of ectopic pregnancy were strongly associated with age; the risk of chlamydial infection decreased with age whereas the risk of ectopic pregnancy increased (both $\mathrm{P}<0.001$ by $\chi^{2}$ test for trend). Among women aged 15-19 years (1990 population: 9027 ) there were 25553 examinations for chlamydia and $1834(7.2 \%)$ positive tests, but there were only 2831 pregnancies of which 18 were ectopic.

Figure 1 shows the trends in the rates of chlamydial infection and ectopic pregnancy for each of the four age groups from 1985 to 1995 . Although absolute levels differ between the rates of chlamydial infection and the rates of ectopic pregnancy, and between age groups, there is a decreasing trend in both rates among the women in three younger age groups (20-24, 25-29, 30-34). Women aged 35-39 years had the highest rates

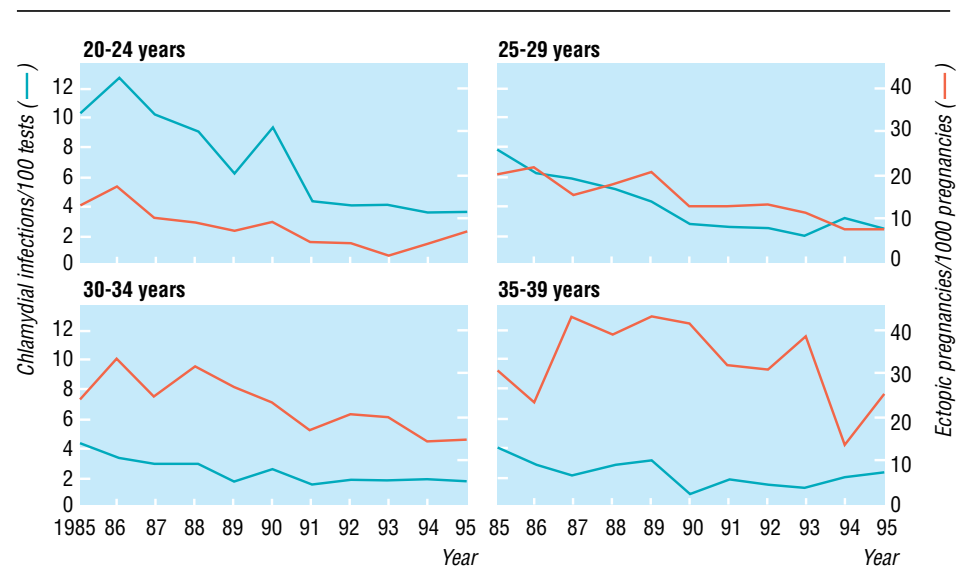

Fig 1 Trends in the rate of chlamydial infection per 100 tests and in the rate of ectopic pregnancy per 1000 pregnancies among women aged 20 to 39 in Uppsala, Sweden, 1985-95

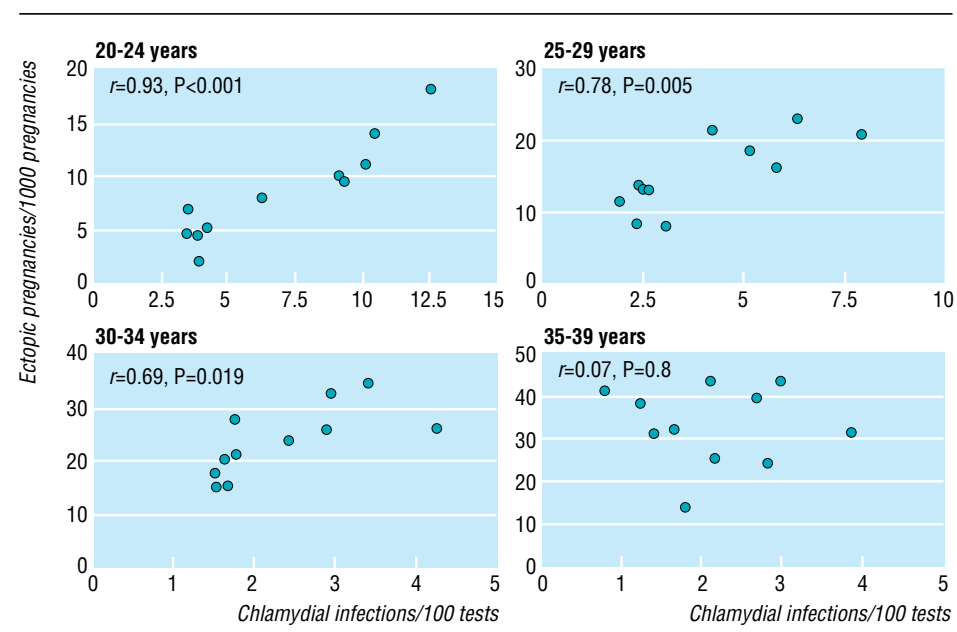

Fig 2 Scatter plot of rate of genital chlamydial infection per 100 tests by the rate of ectopic pregnancy per 1000 pregnancies among women aged 20 to 39 in Uppsala, Sweden, 1985-95 
Table 2 Results of Poisson regression analysis of the association between contemporaneous and earlier rates of genital chlamydial infection with rates of ectopic pregnancy by age group among women in Uppsala, Sweden, 1985-95. Rate ratios (95\% confidence intervals) estimate the effect of an increase of five positive test results per 100 tests on the rate of chlamydial infection

\begin{tabular}{lcccc}
$\begin{array}{l}\text { Rate of } \\
\text { chlamydial } \\
\text { infection }\end{array}$ & $\mathbf{2 0 - 2 4}$ & $\mathbf{2 5 - 2 9}$ & $\mathbf{3 0 - 3 4}$ & $\mathbf{3 5 - 3 9}$ \\
\cline { 2 - 5 } & $1.85(1.44$ to 2.38$)$ & $1.64(1.24$ to 2.17$)$ & $1.11(0.73$ to 1.67$)$ & $0.82(0.51$ to 1.32$)$ \\
\hline In same year & $1.67(1.29$ to 2.15$)$ & $1.72(1.33$ to 2.22$)$ & $2.27(1.53$ to 3.37$)$ & $1.24(0.76$ to 2.04$)$ \\
\hline 1 year earlier & 1.64 group \\
\hline 2 years earlier & $1.46(1.13$ to 1.89$)$ & $1.56(1.22$ to 2.00$)$ & $1.82(1.25$ to 2.66$)$ & $2.58(1.45$ to 4.60$)$ \\
\hline 3 years earlier & $1.34(1.02$ to 1.76$)$ & $1.64(1.27$ to 2.12$)$ & $1.41(0.96$ to 2.06$)$ & $1.55(0.89$ to 2.70$)$ \\
\hline y years earlier & $1.21(0.91$ to 1.62$)$ & $1.71(1.30$ to 2.23$)$ & $1.83(1.21$ to 2.76$)$ & $1.19(0.68$ to 2.08$)$ \\
\hline y years earlier & $1.33(0.97$ to 1.82$)$ & $1.49(1.09$ to 2.03$)$ & $1.70(1.10$ to 2.61$)$ & $1.31(0.66$ to 2.58$)$ \\
\hline
\end{tabular}
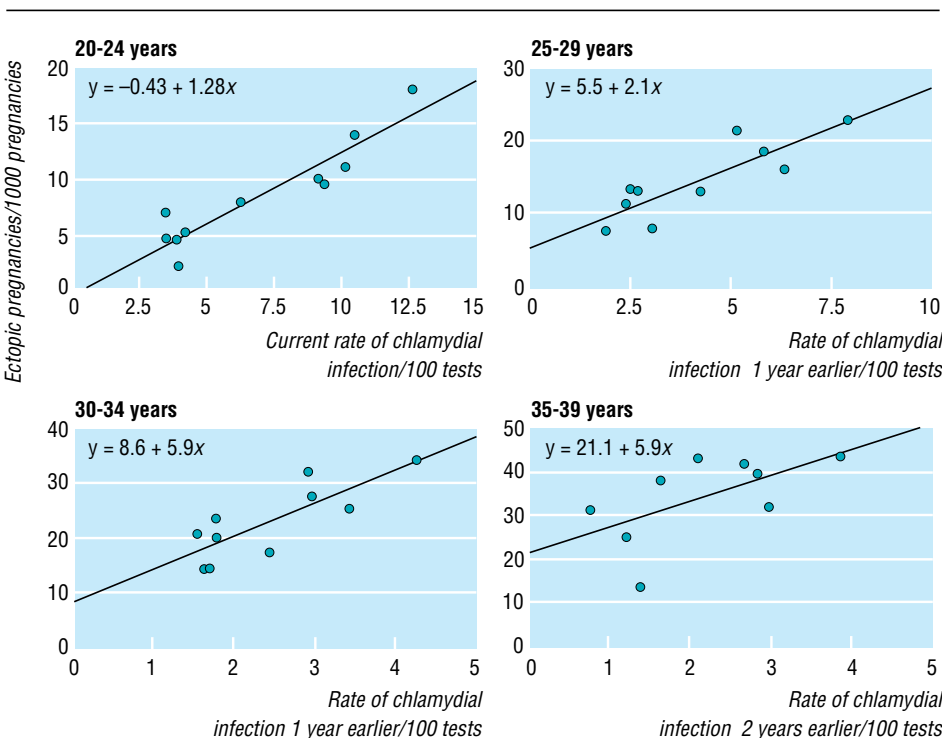

Fig 3 Linear regression analysis of the rate of chlamydial infection per 100 tests by the rate of ectopic pregnancy per 1000 pregnancies among women aged 20 to 39 in Uppsala, Sweden, 1985-95. For the rates of chlamydial infection the time lag which showed the strongest association in the Poisson regression analysis is shown

of ectopic pregnancy with no clear trend over time. There is a strong and highly significant correlation between the rates of chlamydial infection and ectopic pregnancy for women aged 20-24 years $(r=0.93$, $\mathrm{P}<0.001$ ) (fig 2). This correlation gets weaker when women aged 25-29 and 30-34 are analysed; it is not evident among women aged 35-39. The proportion of the variation in the rates of ectopic pregnancy which is explained by chlamydial infections diagnosed in the same year $\left(r^{2}\right)$ was $86 \%$ for women aged 20-24 years, $61 \%$ for women aged $25-29$ years, $48 \%$ for those aged $30-34$ years, and $0 \%$ for women aged $35-39$ years.

Poisson regression analyses were used to explore the association between the risk of ectopic pregnancy with contemporaneous and earlier rates of chlamydial infection (table 2). For all time lag periods there was evidence that associations between rates of chlamydial infection and the risk of ectopic pregnancy varied according to age $(\mathrm{P}<0.05$ to $\mathrm{P}<0.001$ by test of interaction). Separate analyses were therefore performed for women aged 20-24, 25-29, 30-34, and 35-39 years.

The strongest association between ectopic pregnancy and the rate of genital chlamydial infection diagnosed in the same year is observed among women aged 20-24: an increase of five positive results per 100 tests is associated with an $85 \%$ increase in the rate of ectopic pregnancy (rate ratio $1.85, \mathrm{P}<0.001$ ). Significant associations $(\mathrm{P}<0.05)$ also occur for rates of chlamydial infections prevalent 1 to 3 years earlier.

Significant associations $(\mathrm{P}<0.05)$ are observed for the concurrent rate and for all time lags among women aged 25-29. The strength of the association is similar for all rates but the best fit is obtained with the rates occurring 1 year earlier (rate ratio 1.72, $\mathrm{P}<0.001$ ). For women aged 30-34 there is no association between ectopic pregnancy and chlamydial infections diagnosed in the same year (rate ratio $1.11, \mathrm{P}=0.6$ ). Also in this age group the strongest association was evident with rates occurring 1 year earlier (rate ratio 2.27, $\mathrm{P}<$ 0.001 ). Among women aged 35-39 the only significant association that emerged was with rates occurring 2 years earlier (rate ratio $2.58, \mathrm{P}<0.001$ ).

Figure 3 shows scatter plots and linear regression analyses of the rates which showed the strongest association in Poisson regression. The slopes of the regression lines are roughly similar. However, in the group aged 20-24 the line intercepts the $y$ axis near the origin, but the intercept is shifted upwards when older age groups are analysed. Correlation coefficients $(r)$ were 0.93 for the group aged 20-24, 0.85 for the group aged 25-29, 0.81 for the group aged 30-34, and 0.59 for the group aged $35-39$.

\section{Discussion}

The intensive screening effort implemented in Uppsala enabled us to obtain estimates of the rates of chlamydial infection and to examine ecological associations between genital chlamydial infection and ectopic pregnancies. Trends over the study period, 1985 to 1995, showed marked reductions in the rate of chlamydial infections. The concomitant decline in the incidence of ectopic pregnancy suggests that the control programme has been effective in reducing the risk of an important consequence of genital chlamydial infections.

\section{The importance of age}

Regression analyses of the association between the risk of ectopic pregnancy and contemporaneous and earlier rates of chlamydial infection suggest that the association varies systematically with age. Contrary to previous reports, ${ }^{45}$ trends in the risk of ectopic pregnancy do not necessarily lag behind those of chlamydial infections. Among women aged 20-24 a strong association with the concurrent rate of chlamydial infection emerged, which explained $86 \%$ of the variation in the ectopic pregnancy rate. The mechanisms resulting in occlusion of the fallopian tubes remain unknown but it has been suggested that long term processes, including repeated infections, are involved. ${ }^{13}$ Our results show that among these women a large proportion of ectopic pregnancies are a consequence of recent infections and that damage to tubal function resulting from inflammation and the loss of cilia may be present at an early stage. In the same age group (20-24) ectopic pregnancy rates were also associated with the rate of chlamydial infection 1 to 3 years earlier, although the associations tended to be weaker. This may reflect the effect of tubal damage from earlier 
infections and continuing immune mediated destruction.

Among older women (25-39), associations were stronger with the rates of chlamydial infection occurring 1 or more years earlier. In this age group chlamydial infections explained less of the variation in the rates of ectopic pregnancy. The sequelae from gonococcal infections occurring many years earlier could play an additional role. Gonorrhoea was a prevalent cause of salpingitis in the 1960s and 1970s, ${ }^{9}$ when the older women in our study were at their highest risk of acquiring sexually transmitted infections. Since then gonorrhoea has virtually been eradicated and the rate of chlamydial infections has fallen in Uppsala. Further decreases in the rates of ectopic pregnancy are therefore likely to occur in this age group in the future. Finally, factors not related to sexually transmitted infections such as endogenous infections and poor motility in the fallopian tubes might account for a considerable proportion of the ectopic pregnancies occurring in older women. ${ }^{14}$

\section{Limitations of ecological studies}

Findings from ecological analyses must be interpreted with caution. The analyses examine correlations between an exposure (chlamydial infection) and an outcome (ectopic pregnancy) which have been measured at a group rather than an individual level. One problem is that the associations may be confounded by factors that have not been measured. Trends in the prevalence of pelvic inflammatory disease associated with gonorrhoea and in the use of intrauterine contraceptive devices ${ }^{15}$ may have influenced our results. In Uppsala, and elsewhere in Sweden, pelvic inflammatory disease associated with gonorrhoea was very rare during the period studied. ${ }^{9}$

The use of intrauterine devices increases the risk of an ectopic implantation, if implantation occurs, while greatly reducing the probability of any pregnancy occurring. ${ }^{16}$ An increasing use of intrauterine devices would thus reduce the absolute number of ectopic pregnancies and reduce the rate of ectopic pregnancy for every 1000 woman years but there would be an increase in the same rate when expressed for every 1000 pregnancies. In Uppsala there was a decline (of about $45 \%$ ) both in the ectopic pregnancy rate for every 1000 woman years and in the rate for every 1000 pregnancies. It is therefore unlikely that changes in either gonococcal infections or contraceptive methods influenced our findings.

The introduction of more sensitive diagnostic methods, such as serological tests and vaginal ultrasound imaging, could mask a decline in the rate of ectopic pregnancy or could lead to a spurious increase if ectopic pregnancies are diagnosed, which would otherwise have resolved spontaneously without detection. ${ }^{17}{ }^{18}$ In Uppsala, no important changes in diagnostic methods occurred during the study period. In vitro fertilisation and other infertility treatments may lead to a real increase in the rate of ectopic pregnancies, but the increase caused by such treatments has been found to be small. ${ }^{17}$

Results from ecological analyses may not be reproducible at the individual level. ${ }^{19}{ }^{20}$ Direct evidence of the benefits of screening for chlamydia on the incidence of its complications has been found in a con-
Key messages

- The effect of measures to diagnose and treat genital chlamydial infections on the risk of ectopic pregnancy was examined in a county in Sweden

- Rates of chlamydial infection and ectopic pregnancy both declined between 1985 and 1995

- Chlamydial infections seem to be the main cause of ectopic pregnancy in young women; the risk of ectopic pregnancy decreased concurrently with the decline in chlamydial infections

- In older women the associations were less pronounced

trolled trial, in which the risk of pelvic inflammatory disease was reduced among women offered screening. ${ }^{21}$ Our results are also in accordance with observations from other settings. In the United States reductions in the prevalence of chlamydial infections and in the number of hospitalisations for pelvic inflammatory disease and ectopic pregnancy have been reported to coincide with the implementation of programmes to screen for and treat chlamydial infections. ${ }^{22}{ }^{23}$ Conversely, in the South East Thames region in England the rate of ectopic pregnancy increased from 1988 to 1995 (unpublished data). Routine testing in England is consistently practised only in departments of genitourinary medicine, in contrast with the widespread screening for chlamydia in Sweden. Only about $7 \%$ of women in London aged $16-59$ and only $2 \%$ in the remainder of the South East Thames region reported attending genitourinary medicine clinic in the last 5 years ${ }^{24}$ compared with up to $22 \%$ of women aged $15-44$ years tested annually for chlamydia in Uppsala.

\section{Conclusions}

This study has shown that declining rates of chlamydial infection among women in Uppsala during a period of intensive screening, treatment, and contact tracing have been accompanied by a fall in the risk of ectopic pregnancy. Both the importance of chlamydial infections as a cause of ectopic pregnancy and the timing of the decline in the rates of ectopic pregnancy appear to vary with age. Among young women chlamydial infections seem to be the main cause of ectopic pregnancy. Falling rates of chlamydial infections in this group have been accompanied by an immediate reduction in the risk of ectopic pregnancy.

We thank Jonathan Sterne for statistical advice and Gunnar Medin for excellent data management. The Department of Social Medicine at the University of Bristol is part of the MRC Health Services Research Collaboration.

Contributors: ME designed the study, performed the statistical analyses, and wrote the first draft of the paper. $\mathrm{BH}$ was involved in developing the chlamydia database and contributed to writing the paper. NL and BL were involved in writing the paper. GDS contributed to the statistical analyses and writing of the paper. $\mathrm{ME}$ and $\mathrm{BH}$ are guarantors of the study.

Funding: ME is supported by the Swiss National Research Foundation.

Conflict of interest: None. 
1 Mårdh P-A, Ripa T, Svensson L, Weström L. Chlamydia trachomatis infection in patients with acute salpingitis. N EnglJ Med 1977;296:1377-9.

2 Weström L. Incidence, prevalence, and trends of acute pelvic inflammatory disease and its consequences in industrialized countries. Am J Obstet Gynecol 1980;138:880-92.

3 Padian NS, Washington E. Pelvic inflammatory disease: a brief overview. Ann Epidemiol 1994;4:128-32.

4 Chow N, Yonekura ML, Richwald GA, Greenland S, Sweet RL, Schachter $\mathrm{J}$. The association between Chlamydia trachomatis and ectopic pregnancy: a matched-pair, case-control study. JAMA 1990;263:3164-7.

5 Coste J, Laumon B, Brémond A, Collet P, Job-Spira N. Sexually transmitted diseases as major causes of ectopic pregnancy: results from a large case-control study in France. Fertil Steril 1994;62:289-95.

6 Taylor-Robinson D. Chlamydia trachomatis and sexually transmitted disease. $B M J$ 1994:308:150-1.

7 National Board of Health and Welfare. Anonymous Chlamydial infection-preventive measures. General recommendations from the National Board of Health and Welfare. Stockholm: National Board of Health and Welfare, 1990

8 Herrmann B, Egger M. Genital Chlamydia trachomatis infections in Uppsala county, Sweden 1985-1993: declining rates for how much longer? Sex Transm Dis 1995;22:253-60.

9 Kamwendo F, Forslin L, Bodin L, Danielsson D. Decreasing incidences of gonorrhoea- and chlamydia-associated acute pelvic inflammatory disease: a 25-year study from an urban area of central Sweden. Sex Transm Dis 1995;23:384-91.

10 Control of Infectious Diseases Act 1988. Stockholm: Ministry of Health, 1988: 1472. [Swedish code of law.]

11 Robinson AJ, Greenhouse P. Prevention of recurrent pelvic infection by contact tracing: a commonsense approach. $\mathrm{Br} J$ Obstet Gynaecol 1996;103:859-61.

12 Ripa KT, Mårdh P-A. Cultivation of Chlamydia trachomatis in cycloheximide treated McCoy cells. J Clin Microbiol 1977;6:328-31.
13 Cates W, Wasserheit JN. Genital chlamydial infections: epidemiology and reproductive sequelae. Am J Obstet Gynecol 1991;164:1771-81.

14 Cates W, Rolfs RT, Aral SO. Sexually transmitted diseases, pelvic inflammatory disease, and infertility: an epidemiologic update. Epidemiol Rev $1990 ; 12: 199-221$.

15 Xiong X, Buekens P, Wollast E. IUD use and the risk of ectopic pregnancy: a meta-analysis of case-control studies. Contraception 1995;52:23-34.

16 Franks AL, Beral V, Cates W, Hogue CJR. Contraception and ectopic pregnancy risk. Am J Obstet Gynecol 1990;163:1120-3.

17 Skjeldestad FE, Kendrick JS, Atrash HK, Daltveit AK. Increasing incidence of ectopic pregnancy in one Norwegian county: a population based study, 1970-1993. Acta Obstet Gynecol Scand 1997;76:159-65.

18 Chow W-H, Daling JR, Cates W, Greenberg RS. Epidemiology of ectopic pregnancy. Epidemiol Rev 1987;9:70-94.

19 Piantadosi S, Byar DP, Green SB. The ecological fallacy. Am J Epidemiol 1988; $127: 893-904$

20 Susser M. The logic in ecological. I. The logic of analysis. Am J Pub Health 1994;84:825-9.

21 Scholes D, Stergachis A, Heidrich FE, Andrilla H, Holmes KK, Stamm WE. Prevention of pelvic inflammatory disease by screening for cervical chlamydia infection. N Engl J Med 1996;334:1362-6.

22 Hillis SD, Nakashima A, Amsterdam L, Pfister J, Vaughn M, Addiss D, et al. The impact of a comprehensive chlamydia prevention program in Wisconsin. Fam Plann Perspect 1995;27:108-11.

23 Addiss DG, Vaughin ML, Ludka D, Pfister J, Davis JP. Decreased prevalence of Chlamydia trachomatis infection associated with a selective screening program in family planning clinics in Wisconsin. Sex Transm Dis 1993;20:28-34.

24 Johnson AM, Wadsworth J, Wellings K, Field J. Sexual attitudes and lifestyles. Oxford: Blackwell Scientific, 1994.

(Accepted 12 February 1998)

\title{
Blood pressure and mortality in elderly people aged 85 and older: community based study
}

\author{
Hendriek C Boshuizen, Gerbrand J Izaks, Stef van Buuren, Gerard J Ligthart
}

\section{TNO Prevention and Health, Division of Public Health and \\ Prevention, Leiden, \\ Netherlands \\ Hendriek C \\ Boshuizen, \\ epidemiologist \\ Stef van Buuren, \\ statistician \\ Section of \\ Gerontology and \\ Geriatrics, \\ Department of \\ General Internal \\ Medicine, Leiden \\ University Medical \\ Centre, Leiden \\ Gerbrand J Izaks, research fellow \\ Gerard J Ligthart, \\ associate professor \\ Correspondence to: \\ Dr Boshuizen \\ HC.Boshuizen@ \\ PG.TNO.NL}

BMJ 1998;316:1780-4

\begin{abstract}
Objective: To determine whether the inverse relation between blood pressure and all cause mortality in elderly people over 85 years of age can be explained by adjusting for health status, and to determine whether high blood pressure is a risk factor for mortality when the effects of poor health are accounted for.

Design: 5 to 7 year follow up of community residents aged 85 years and older.

Setting: Leiden, the Netherlands.

Subjects: 835 subjects whose blood pressure was recorded between 1987 and 1989.

Main outcome measure: All cause mortality.

Results: An inverse relation between blood pressure and all cause mortality was observed. For diastolic blood pressure crude 5 year all cause mortality decreased from $88 \%$ (52/59) $(95 \%$ confidence interval $79 \%$ to $95 \%$ ) in those with diastolic blood pressures $<65 \mathrm{~mm} \mathrm{Hg}$ to $59 \%(27 / 46)(44 \%$ to $72 \%)$ in those with diastolic pressures $>100 \mathrm{~mm} \mathrm{Hg}$. For systolic blood pressure crude 5 year all cause mortality decreased from $85 \%(95 / 112)(78 \%$ to $91 \%)$ in those with systolic pressures < $125 \mathrm{~mm} \mathrm{Hg}$ to $59 \%(13 / 22)$ (38\% to $78 \%$ ) in those with systolic pressures $>200 \mathrm{~mm} \mathrm{Hg}$. This decrease was no longer significant after adjustment for indicators of poor health. No relation existed between blood pressure and mortality from cardiovascular causes or stroke after adjustment for age and sex, but after adjustment for age, sex, and indicators of poor health there was a
\end{abstract}

positive relation between diastolic blood pressure and mortality from both cardiovascular causes and stroke. Conclusion: The inverse relation between blood pressure and all cause mortality in elderly people over 85 is associated with health status.

\section{Introduction}

In western societies blood pressure rises with age. It is not clear if this is the result of a pathological process that needs treatment or if it is part of the natural ageing process. Treating hypertension helps prevent stroke and coronary heart disease in middle aged and elderly people younger than 80. In two trials, however, no benefit of treatment was observed in subjects aged 80 and older. $^{12}$ Treating isolated systolic hypertension does not reduce all cause mortality in this age group, although it prevents stroke and heart failure. ${ }^{34}$

Since the number of subjects aged 80 and older included in these trials is small, evidence from observational studies may help in deciding whether to treat hypertension in patients at this age. Previous observational studies comprised of people aged 80 and older have found no relation between blood pressure and mortality, ${ }^{5}$ and a U shaped relation, ${ }^{6}$ or even an inverse relation in which higher blood pressure was associated with lower mortality. ${ }^{7-9}$ However, adjustment for confounding factors was made in only one study ${ }^{9}$; in this study only 35 deaths occurred in those aged over 80 .

To determine whether the relation between blood pressure and all cause mortality differs in elderly people older than 85 as compared with younger 